\title{
Complexity in Manufacturing Processes and Systems 2019
}

\author{
Rosario Domingo $\mathbb{D}^{1},{ }^{1}$ Julio Blanco-Fernández $\mathbb{D}^{2},{ }^{2}$ and Jorge Luis García-Alcaraz $\mathbb{D}^{3}$ \\ ${ }^{1}$ Universidad Nacional de Educación a Distancia (UNED), Madrid, Spain \\ ${ }^{2}$ Universidad de La Rioja, Logroño, Spain \\ ${ }^{3}$ Autonomous University of Ciudad Juarez, Ciudad Juárez, Mexico \\ Correspondence should be addressed to Rosario Domingo; rdomingo@ind.uned.es
}

Received 2 December 2019; Accepted 2 December 2019; Published 3 July 2020

Copyright ( $) 2020$ Rosario Domingo et al. This is an open access article distributed under the Creative Commons Attribution License, which permits unrestricted use, distribution, and reproduction in any medium, provided the original work is properly cited.

This Special Issue is continuation of the work published in 2018 with the same name [1]. As is expected, the control or behavior knowledge of factors that influence on the production performance continues to be important in the academic and industrial field. Thus, new algorithms are developed to reduce the uncertainty and improve the efficiency in machines [2], processes [3,4], and even the supply chain [5], and new methods are proposed to know the relationship among production factors in some sectors $[6,7]$ or more specific as in assembly lines [8]. In this context, the communication technologies and the advanced manufacturing technologies could be considered because they affect the performance and facilitate to monitoring the production process $[9,10]$ or new customized product configurations [11].

Fourteen papers have been accepted for this special issue, after a strict review process. Between them, there are four papers devoted to the manufacturing process, and almost all of them show methods to control some manufacturing parameters.

V. Flores and B. Keith in their paper titled "Gradient Boosted Trees Predictive Models for Surface Roughness in High-Speed Milling in the Steel and Aluminum Metalworking Industry" present a model of the surface roughness average that evaluates the influence of the cutting conditions in the machining process, in particular, in high-speed endmilling. The model is constructed after a predictive analysis by gradient boosted trees derivate of machine learning techniques.

M. Du et al. in their paper titled "Intelligent Turning Tool Monitoring with Neural Network Adaptive Learning" investigate the tool state monitoring in the machining process using artificial intelligent techniques. The paper has an experimental procedure applied to inserts in the turning process, whereby the data are taken using vibration and acoustic emission signals for tool monitoring. The data, taken in real time, are treated by multitheory fusion of wavelet decomposition and the ReliefF algorithm and after neural network adaptive learning. The results indicate a good prediction.

M. Jiménez et al. in their paper titled "Additive Manufacturing Technologies: An Overview about 3D Printing Methods and Future Prospects" realize an overview related to additive manufacturing and considering the structural design of the machines, possible alternatives, more significant materials, technological limitations, and new perspectives in professional and academic fields.

T. R. Gazizov et al. devoted the paper "Solving the Complexity Problem in the Electronics Production Process by Reducing the Sensitivity of Transmission Line Characteristics to Their Parameter Variations" to the electronics production process. With a versatile approach, the study analyzes the reduction in sensitivity of transmission line characteristics to their parameter variations. Different results in microstrip lines are presented.

The other group of papers has a more comprehensive approach, and they consider external factors to manufacturing processes, considering the production system, the supply chain, or the enterprise. They are presented in the following paragraphs.

Q. Chen et al. in their paper titled "Dynamic Matching in Cloud Manufacturing considering Matching Costs" explore 
the resources' dynamic matching in a manufacturing supply chain that operates under a cost-sharing contract. The authors model the evolution of resource sharing by means of differential equations, study the optimal matching strategies by a two-stage differential game based on the dynamic control approach, and design a cost-sharing contract to coordinate and improve the supply chain's performance. Moreover, a numerical analysis shows the influence of transaction fee and purchasing costs on different geographical regions and strategies.

Y. Changwei and G. Jinhao focus their paper titled "Research on Warning Threshold Value Optimization and Disposal Strategy of Earthquake Early Warning for Highspeed Railway under Earthquake" on the design of a model to study the derailment phenomenon of trains and the seismic performance of different structural types. A dynamic simulation allows identifying the safe running speed threshold of high-speed trains under earthquake.

F. Chen et al. in their paper titled "An Integrated Metaheuristic Routing Method for Multiple-Block Warehouses with Ultranarrow Aisles and Access Restriction" develop a routing algorithm that allows integrating an ant colony optimization (ACO) and integer-coded genetic algorithm (GA). This algorithm is applied to internal logistic with ultranarrow aisles and access restrictions. A simulation considering 12 warehouse layouts shows that the new algorithm allows better solutions according to certain efficiency indexes.

J. Qin and Z. Li in their paper titled "Reliability and Sensitivity Analysis Method for a Multistate System with Common Cause Failure" develop a reliability assessment method for a multistate system (MSS) with series-parallel and common cause failure (CCF). The method is based on the universal generating function (UGF) to which is incorporated the CCF. Different applications show the goodness of this method by means of calculus of reliability indexes.

The paper "Pricing and Collection Rate for Remanufacturing Industry considering Capacity Constraint in Recycling Channels" written by L. Xu et al. explores the coordination in a closed-loop supply chain taking into account capacity constraints in recycling channels. The paper considers two scenarios, centralized and decentralized, to evaluate the equilibriums of decisions and profits, according to the game theory. Findings indicate that, in recycling channels, when the capacity constraints surpass a threshold, the profit is modified and that these constraints affect the selling price more than other variables.

F. Brocal et al. in their paper titled "Emerging Risk Management in Industry 4.0: An Approach to Improve Organizational and Human Performance in the Complex Systems" propose an organizational and human performance approach to improve the emerging risk management in systems such as Human-Machine Interactions (HMI) and Human-Robot Interaction (HRI). The concept of emerging risk management in this framework is introduced and its effects on complex production systems.

The paper "The Incentive Model in Supply Chain with Trade Credit and Default Risk" written by H. Cheng et al. is devoted to trade credit analysis in supply chain management with default risk and moral hazard. The authors develop the principal-agent models by means of incentive theory in a newsvendor approach and considering situations of symmetric and asymmetric information. Findings indicate that with an incentive model, the order quantity is lowered in the incentive contract to mitigate the effect of moral hazard.

$\mathrm{X}$. Wang et al. in their paper titled "A Novel MOEA/D for Multiobjective Scheduling of Flexible Manufacturing Systems" present a deadlock-free multiobjective evolutionary algorithm based on decomposition (DMOEA/D) applied to flexible manufacturing systems. This algorithm decomposes a multiobjective scheduling problem in single-objective subproblems and can resolve all these subproblems in a single run. The computational results show that this method finds better Pareto solutions than other traditional approaches.

P. Urgilés et al. in their paper titled "Analysis of the Earned Value Management and Earned Schedule Techniques in Complex Hydroelectric Power Production Projects: Cost and Time Forecast" focus on the uncertainty of industrial projects. The paper analyzes the efficiency of the Earned Value Management technique, as well as its Earned Schedule extension. Simulation indicates that cost forecasting can be predicted very accurately; however, the duration forecasting is not accurate.

The paper "STEAM-ME: A Novel Model for Successful Kaizen Implementation and Sustainable Performance of SMEs in Vietnam" written by T. L. Nguyen proposes a model that allows identifying critical factors during the implementation and sustainable performance of Kaizen concept in small and medium enterprises (SMEs). This model named "STEAM-ME" is created from a survey carried out in 213 participants from 62 SMEs; this survey allows identifying seven factors after an exploratory statistical analysis and structural equation modeling: supports from senior management, training, environment, assessment, motivation, mindset, and engagement of all members in the organization.

This special issue, as the first issue dedicated to Complexity in Manufacturing Processes and Systems, can be interesting for engineers and managers with responsability in manufacturing plants, where new methods and results show how the complexity can be managed in industrial environments. New advances can be made from the findings of these papers.

\section{Conflicts of Interest}

The editors declare that there are no conflicts of interest regarding the publication of this special issue.

\section{Acknowledgments}

The Guest Editorial Team would like to thank all the authors for their interest in selecting the special issue "Complexity in Manufacturing Processes and Systems 2019" and for their valuable contributions.

Rosario Domingo Julio Blanco-Fernández Jorge Luis García-Alcaraz 


\section{References}

[1] R. Domingo, J. Blanco-Fernández, J. L. García-Alcaraz, and L. Rivera, "Complexity in manufacturing processes and systems," Complexity, vol. 2018, Article ID 8738764, 3 pages, 2018.

[2] R. Calvo, R. D’Amato, E. Gómez, and R. Domingo, "Integration of error compensation of coordinate measuring machines into feature measurement: part I-model development," Sensors, vol. 16, no. 10, p. 1610, 2016.

[3] C. Garriz and R. Domingo, "Development of trajectories through the kalman algorithm and application to an industrial robot in the automotive industry," IEEE Access, vol. 7, pp. 23570-23578, 2019.

[4] M. Oropesa Vento, J. L. García Alcaraz, A. A. Maldonado Macías, and V. Martínez Loya, "The impact of managerial commitment and Kaizen benefits on companies," Journal of Manufacturing Technology Management, vol. 27, no. 5, pp. 692-712, 2016.

[5] S. Kusi-Sarpong, H. Gupta, and J. Sarkis, "A supply chain sustainability innovation framework and evaluation methodology," International Journal of Production Research, vol. 57, no. 7, pp. 1990-2008, 2019.

[6] J. R. Díaz-Reza, J. R. Mendoza-Fong, J. Blanco-Fernández, J. A. Marmolejo-Saucedo, and J. L. García-Alcaraz, “The role of advanced manufacturing technologies in production process performance: a causal model," Applied Sciences, vol. 9, no. 18 , p. $3741,2019$.

[7] R. Villanueva-Ponce, J. L. Garcia-Alcaraz, G. Cortes-Robles, J. Romero-Gonzalez, E. Jiménez-Macías, and J. BlancoFernández, "Impact of suppliers' green attributes in corporate image and financial profit: case maquiladora industry," The International Journal of Advanced Manufacturing Technology, vol. 80, no. 5-8, pp. 1277-1296, 2015.

[8] V. G. Cannas, M. Pero, R. Pozzi, and T. Rossi, "Complexity reduction and kaizen events to balance manual assembly lines: an application in the field," International Journal of Production Research, vol. 56, no. 11, pp. 3914-3931, 2018.

[9] J. L. García-Alcaraz, V. Martínez-Loya, J. R. Díaz-Reza, J. Blanco-Fernández, E. Jiménez-Macías, and A. J. G. López, "Effect of ICT integration on SC flexibility, agility and company' performance: the Mexican maquiladora experience," Wireless Networks, 2019.

[10] N. F. Alkayem, M. Cao, and M. Ragulskis, "Damage diagnosis in $3 \mathrm{D}$ structures using a novel hybrid multiobjective optimization and FE model updating framework," Complexity, vol. 2018, Article ID 3541676, 13 pages, 2018.

[11] S. Bednar and E. Rauch, "Modeling and application of configuration complexity scale: concept for customized production," The International Journal of Advanced Manufacturing Technology, vol. 100, no. 1-4, pp. 485-501, 2019. 